Migrationsforschung in Deutschland 
Débora Maehler • Ulrich Schmidt-Denter

\section{Migrationsforschung in Deutschland}

Leitfaden und Messinstrumente zur Erfassung psychologischer Konstrukte

Springer VS 
Débora Maehler, GESIS - Leibniz-Institut

für Sozialwissenschaften, Mannheim, Deutschland
Ulrich Schmidt-Denter

Universität zu Köln,

Köln, Deutschland

ISBN 978-3-531-19244-4

ISBN 978-3-531-19245-1 (eBook)

DOI 10.1007/978-3-531-19245-1

Die Deutsche Nationalbibliothek verzeichnet diese Publikation in der Deutschen Nationalbibliografie; detaillierte bibliografische Daten sind im Internet über http://dnb.d-nb.de abrufbar.

\section{Springer VS}

() Springer Fachmedien Wiesbaden 2013

Das Werk einschließlich aller seiner Teile ist urheberrechtlich geschützt. Jede Verwertung, die nicht ausdrücklich vom Urheberrechtsgesetz zugelassen ist, bedarf der vorherigen $\mathrm{Zu}-$ stimmung des Verlags. Das gilt insbesondere für Vervielfältigungen, Bearbeitungen, Übersetzungen, Mikroverfilmungen und die Einspeicherung und Verarbeitung in elektronischen Systemen.

Die Wiedergabe von Gebrauchsnamen, Handelsnamen, Warenbezeichnungen usw. in diesem Werk berechtigt auch ohne besondere Kennzeichnung nicht zu der Annahme, dass solche Namen im Sinne der Warenzeichen- und Markenschutz-Gesetzgebung als frei zu betrachten wären und daher von jedermann benutzt werden dürften.

Gedruckt auf säurefreiem und chlorfrei gebleichtem Papier

Springer VS ist eine Marke von Springer DE. Springer DE ist Teil der Fachverlagsgruppe Springer Science+Business Media.

www.springer-vs.de 


\section{Danksagung}

Die Stichprobengewinnung sowie die gesamte Durchführung des Projekts, aus dem die hier präsentierten Skalen stammen, war nur mit Hilfe eines engagierten und motivierten Teams möglich. Ganz herzlichen Dank gebührt den hier in alphabetischer Reihenfolge aufgeführten ehemaligen Mitarbeitern: AnnaLinda Balkowski, Jana Bergmann, Marcella Birken, Eda Cetin, Nino Gogodze, Tea Kacharava, Maren Kloosterhuis, Christian Marin, Tatiana Radchenko, Nelly Schwab-Solaymani, Irene Skuballa, Anika Stuppy und Sandra Zerres.

Es ist uns ein Bedürfnis, die Leistung und den Einsatz zweier ehemaliger Mitarbeiterinnen besonders hervorzuheben: Dominika Julkowski und Katharina Pöhls; ihnen stehen großer Dank und Anerkennung zu.

Köln, im Januar 2012

Débora Maehler \& Ulrich Schmidt-Denter 


\section{Inhalt}

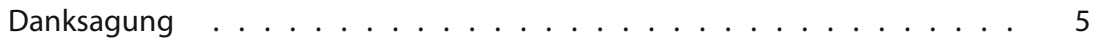

Abbildungsverzeichnis $\ldots \ldots \ldots \ldots$

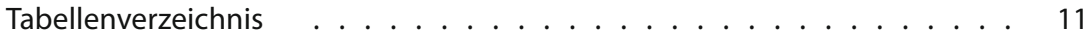

1 Einleitung $\ldots \ldots \ldots \ldots \ldots \ldots$

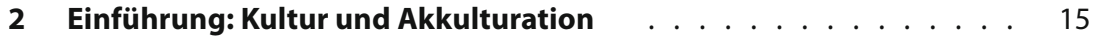

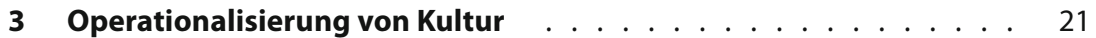

3.1 Kulturelle Dimensionen nach Hofstede . . . . . . . . . . . . . . . . 21

3.2 Kulturelle Dimensionen nach Schwartz _ . . . . . . . . . . . . . 23

3.3 Kulturelle Dimensionen nach Inglehart und Welzel _ . . . . . . . . 30

3.4 Schlussfolgerungen . . . . . . . . . . . . . . 33

4 Typologien in der Akkulturationsforschung $\ldots \ldots \ldots$

4.1 Akkulturationsmodelle und Messmethoden . . . . . . . . . . . . . 37

4.2 Akkulturationsforschung in Deutschland . . . . . . . . . . . . 42

5 Äquivalenz interkultureller Messungen $\ldots \ldots$. . . . . . . . . . . 47

5.1 Störfaktoren und Verzerrungen . . . . . . . . . . . . . . . . 47

5.2 Äquivalenzebenen . . . . . . . . . . . . . . . . . . . 49

5.3 Statistische Analysemethoden . . . . . . . . . . . . . 50

6 Auswahl der Erhebungsmethode $\ldots \ldots \ldots 55$

7 Erfassung des soziodemografischen Hintergrundes _ . . . . . . . 59

7.1 Sprachkenntnisse . . . . . . . . . . . . . . . . . . . 59

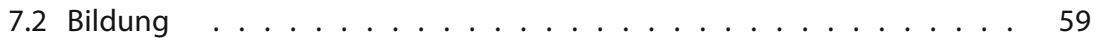

7.3 Sozioökonomischer Status _ . . . . . . . . . . . . . 60 


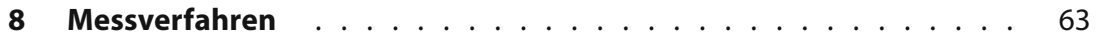

8.1 Individuelle Dispositionen $\ldots \ldots \ldots$

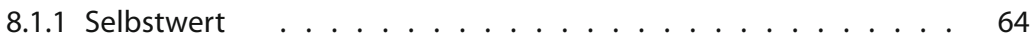

8.1 .2 Big Five . . . . . . . . . . . . . . . . . . 65

8.2 Akkulturation (Orientierung) $\ldots \ldots \ldots$

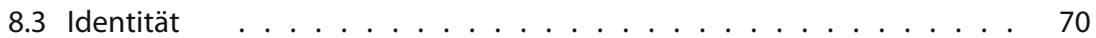

8.4 Kultureller Hintergrund (Wertdimension) _ . . . . . . . . . . 72

8.4.1 Wertvorstellungen: Ansatz von Schwartz . . . . . . . . . 72

8.4.2 Kulturelle Distanz: Ansatz von Hofstede . . . . . . . . . . . . . 74

8.5 Motive der Auswanderung . . . . . . . . . . . . . 76

8.6 Motive der Einbürgerung _ . . . . . . . . . . . . . . . . . 78

9 Zusammenfassung und Ausblick $\ldots \ldots \ldots$

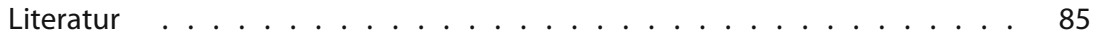

Anhang A:Tabellen . . . . . . . . . . . . . . . . 95

Anhang B: Abbildungen . . . . . . . . . . . . . 125 


\section{Abbildungsverzeichnis}

Abbildung 3.1 Kreisstruktur der zehn individuellen Wertetypen _ . . . . 25

Abbildung 3.2 Einordnung von 76 nationalen Gruppen anhand der sieben Wertedimensionen $\quad \ldots \ldots \ldots$

Abbildung 3.3 Cultural Map of the World . . . . . . . . . . . . . . . 31

Abbildung 3.4 Einteilung der Länder nach den Ergebnissen der Clusteranalyse . . . . . . . . . . . 34

Abbildung 4.1 Überblick über Modelle, Erhebungsund Auswertungsmethoden . . . . . . . . . . . 38

Abbildung B.1 Syntax für Targetrotation . . . . . . . . . . . . . . . 125

Abbildung B.2 Dendrogramm der Wertedimensionen von Inglehart und Welzel ～. . . . . . . . . . . . 128 


\section{Tabellenverzeichnis}

Tabelle 4.1 Auswertungsverfahren und Konzepte

in der Akkulturationsforschung in Deutschland . . . . . . 43

Tabelle 8.1 Itemkennwerte: Selbstwert _ . . . . . . . . . . . . . 65

Tabelle 8.2 Itemkennwerte: Big Five . . . . . . . . . . . . . . 66

Tabelle 8.3 Itemkennwerte: Akkulturationsorientierung deutsche Kultur . . . . . . . . . . . . . . . . . 69

Tabelle 8.4 Itemkennwerte: Akkulturationsorientierung Herkunftskultur . . . . . . . . . . . . . . 69

Tabelle 8.5 Itemkennwerte: Identifikation mit der deutschen Kultur . . . . . . . . . . . . . 71

Tabelle 8.6 Itemkennwerte: Identifikation mit der Herkunftskultur . . . . . . . . . . . . . . . . 71

Tabelle 8.7 Itemkennwerte: Wertvorstellungen _ . . . . . . . . . 73

Tabelle 8.8 Itemkennwerte: Kulturelle Distanz . . . . . . . . . . . . . . 75

Tabelle 8.9 Itemkennwerte: Motive der Auswanderung _ . . . . . . . 77

Tabelle 8.10 Itemkennwerte: Motive der Einbürgerung . . . . . . . . . . 80

Tabelle A.1 Kategorisierung der untersuchten Länder nach den Wertedimensionen von Inglehart . . . . . . . 95

Tabelle A.2 Klassifizierungsergebnisse (Diskriminanzanalyse) . . . . . . 99

Tabelle A.3 Übersicht der Skalenkennwerte . . . . . . . . . . . . . . . 100

Tabelle A.4 Faktorenanalyse, Items Selbstwert ． . . . . . . . . . . . . . 101

Tabelle A.5 Koeffizienten nach Targetrotation, Skala Selbstwert ～. . . . . 102

Tabelle A.6 Faktorenanalyse, Items Big Five . . . . . . . . . . . . . . . 103

Tabelle A.7 Koeffizienten nach Targetrotation, Skala Big Five . . . . . . . 106

Tabelle A.8 Faktorenanalyse, Items Akkulturationsorientierung deutsche Kultur . . . . . . . . . . . . . . . . . 107

Tabelle A.9 Koeffizienten nach Targetrotation, Skala Akkulturationsorientierung deutsche Kultur . . . . . 108

Tabelle A.10 Faktorenanalyse, Items Akkulturationsorientierung Herkunftskultur . . . . . . . . . . . . . . . . 109

Tabelle A.11 Koeffizienten nach Targetrotation, Skala Akkulturationsorientierung Herkunftskultur . . . . . . 110 
Tabelle A.12 Faktorenanalyse, Items Skala Identifikation mit der deutschen Kultur . . . . . . . . . . . . . . . . . . 111

Tabelle A.13 Koeffizienten nach Targetrotation, Skala Identifikation mit der deutschen Kultur . . . . . . . . . . . . . . . . . . 112

Tabelle A.14 Faktorenanalyse, Items Identifikation mit der Herkunftskultur

Tabelle A.15 Koeffizienten nach Targetrotation, Skala Identifikation mit der Herkunftskultur . . . . . . . . . . . . . . . . . . . 114

Tabelle A.16 Faktorenanalyse, Items Wertvorstellungen ～. . . . . . . . . . 115

Tabelle A.17 Koeffizienten nach Targetrotation, Skala Wertvorstellungen . . . . . . . . . . . . . . . . 117

Tabelle A.18 Faktorenanalyse, Items Kulturelle Distanz ～. . . . . . . . . . 118

Tabelle A.19 Koeffizienten nach Targetrotation, Skala Kulturelle Distanz ． . . . . . . . . . . . . . . . . . . 120

Tabelle A.20 Faktorenanalyse, Items Motive der Einbürgerung . . . . . . 121

Tabelle A.21 Koeffizienten nach Targetrotation, Skala Motive der Einbürgerung . . . . . . . . . . . . . 123 